\title{
TRYING TO ENLARGE THE SKY COVERAGE OF THE FAST
}

\author{
SHENGYIN WU ${ }^{1}$, SHOUGUAN WANG ${ }^{1}$, YUKUAN MAO ${ }^{2}$ and YAN SU ${ }^{1}$ \\ ${ }^{1}$ National Astronomical Observatories, Chinese Academy of Sciences \\ ${ }^{2}$ Xidian University, Xi'an, China
}

\begin{abstract}
The proposed FAST project is a novel giant spherical radio telescope, with active elements which form a temporary paraboloid to track radio objects in the sky. Efforts have been made to extend the limited sky coverage that is a characteristic disadvantage of Arecibo-style radio telescopes. Three measures under investigation are introduced in this paper. The expected performance of the telescope is described, and a brief comparison is made with some of the largest existing radio telescopes.
\end{abstract}

\section{Introduction}

Limited sky coverage seems to be one of the most important shortcomings of spherical radio telescopes due to available terrain (specified geometric parameters), intrinsic blockage and/or paraxial focus line. For instance, the Arecibo Radio Telescope has the biggest collecting area among the radio telescopes built to date, but only small sky coverage, up to $20^{\circ}$ from the zenith. This restriction is particularly undesirable for observations where the telescope is required to track a radio source for a long time, as in VLBI imaging.

The planned FAST project involves building a huge spherical radio telescope with active panels. A fraction of the panels can be used to simulate a paraboloid to simplify and lighten the feed and the feed driving system (Qiu 1998). By introducing such new ideas for the traditional spherical radio telescope, and by making attempts to enlarge the sky coverage of the FAST as far as possible, we hope to realize a very powerful instrument in comparison with the current radio telescopes.

\section{Attempts to Expand the Sky Coverage of the FAST}

\subsection{KARST DEPRESSIONS AND THE GEOMETRIC PARAMETERS OF FAST}

There are rich resources of the Karst terrain and depressions in Guizhou province, in the southwest of China. In fact, there are many depressions suitable for the FAST project. A number distribution of essential parameters (number, depth and diameter) of the depressions in two regions of Guizhou province has been determined (Wu and Chen 1996).

Considering possible blocking effects, allowable spillover, feature of terrain, the feasibility of engineering and the maintenance accessibility, the following geo- 


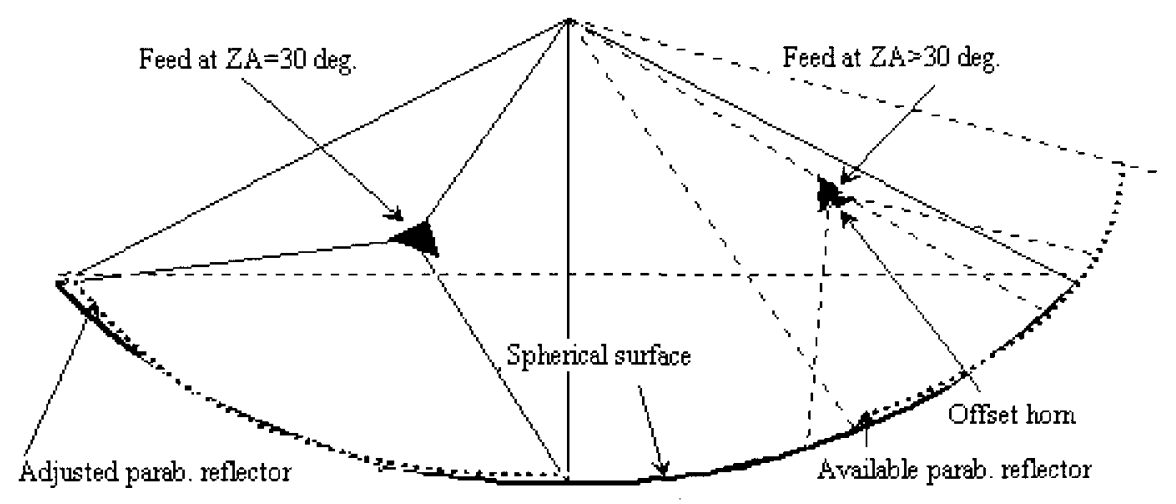

Figure 1. Illustration of the offset illumination of the parabola for the FAST.

metric parameters of the reflector of FAST have been tentatively suggested (Wu, 1999): curvature radius $R=300 \mathrm{~m}$, the opening angle $\theta=120^{\circ}$, hence depth $\mathrm{H}=$ $150 \mathrm{~m}$ and total aperture $\mathrm{D}=520 \mathrm{~m}$.

\subsection{OfFSET ILLUMINATION OF THE SIMULATED APERTURE}

Recent development of radio telescopes has seen array feeds used more and more to realize multi-beams; these developments allow the sky to be scanned more rapidly. For FAST, the $-10 \mathrm{~dB}$ point of the feed would reach the edge of the reflector when the telescope scanned up to the zenith angle of $30^{\circ}$. The available parabolic aperture would become smaller, and change its form from a circle to something like an ellipse, if the telescope were scanned beyond this point (see Figure 1). We must therefore narrow the feed pattern from a circle to an ellipse matched with the available aperture, and keep pointing the feed at the center of the available aperture, to avoid increasing the spillover and ground noise and to improve the beam pattern.

If a feed array was adopted in the FAST, 2 or 3 feed elements could be connected to form a broadside array to achieve 2 or 3 narrowed feed patterns. By offsetting the narrowed beam, we get an offset paraboloid; although the gain is lowered, the telescope will still be a high-gain one.

For a traditional horn feed, the required control of feed inclination with respect to the major optical axis of the paraboloid, the available reception area and equivalent aperture can be calculated exactly ( $\mathrm{Wu}, 1999 \mathrm{~b})$. The results of these calculations, and conclusions drawn from them, provide bases on which to design a special servo system for controlling the inclination of the feed. It has been shown that the available equivalent aperture would be not less than $200 \mathrm{~m}$ even for observations made at zenith angle of $60^{\circ}(\mathrm{Wu}, 2000)$. The equivalent aperture is indicated by the squared-dashed curve in Figure 3 . 


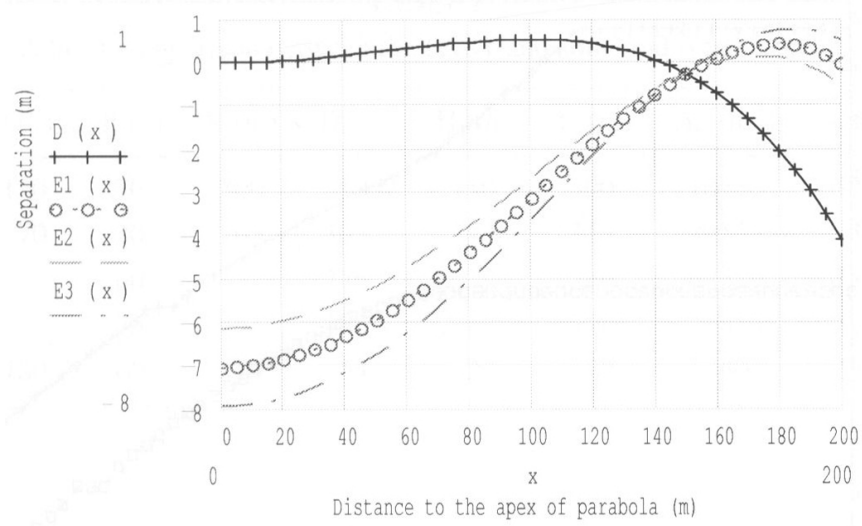

Figure 2. The centripetal discrepancies of an example of inner parabola and outer parabolic ring to the spherical main surface.

\subsection{MULTI-PARABOLA FITTING AND FURTHER EXPANDING OBSERVABLE ZENITH ANGLE BEYOND A HALF OF THE OPENING ANGLE}

The discrepancy between a paraboloid and the spherical surface is small inside an aperture of some 150m, see (Qiu, 1998) and the solid curve in Figure 2, but becomes important outside this aperture. In fact, although the diameter of the spherical cap is $520 \mathrm{~m}$, the diameter of the simulated aperture is only $300 \mathrm{~m}$, so we could fit to a sector ring paraboloid in the remaining part of the cap. In this fitting, the two sectors of paraboloid differ only in respect of small displacements of the apex and the focus. The aim of the proposed multi-parabola fitting concept is to enlarge the utilization factor of the reflector and increase the available aperture at large zenith angles.

Dual-parabola fitting has been modelled in order to test the feasibility of the proposal (Wu, 2000). The results show that we could extend the aperture up to $400 \mathrm{~m}$, under the constraint that the centripetal adjustment required be less than $0.7 \mathrm{~m}$ and matched with that at the edge of the inner aperture of $300 \mathrm{~m}$. Figure 2 indicates one examples of the dual-parabola fitting. If the whole reflector, consisting of the inner aperture and the outer ring together, is used to observe and track the same object, an equivalent aperture can be calculated, and this is shown by the upper curve (crossed) in Figure 3. This shows that an aperture of $210 \mathrm{~m}$ may be available even at the zenith angle of $70^{\circ}$. This should be contrasted with the case of the offset-illuminated inner aperture, which is also shown (squares) in Figure 3. On the other hand we have to pay attention to the technical difficulties associated with assembly and driving of two separate feed systems (some 15 meters apart), and those associated with the variation of the efficiency and gain of the telescope vs. the zenith angle. In reality, there can be many possible ways to use the remaining surface. 


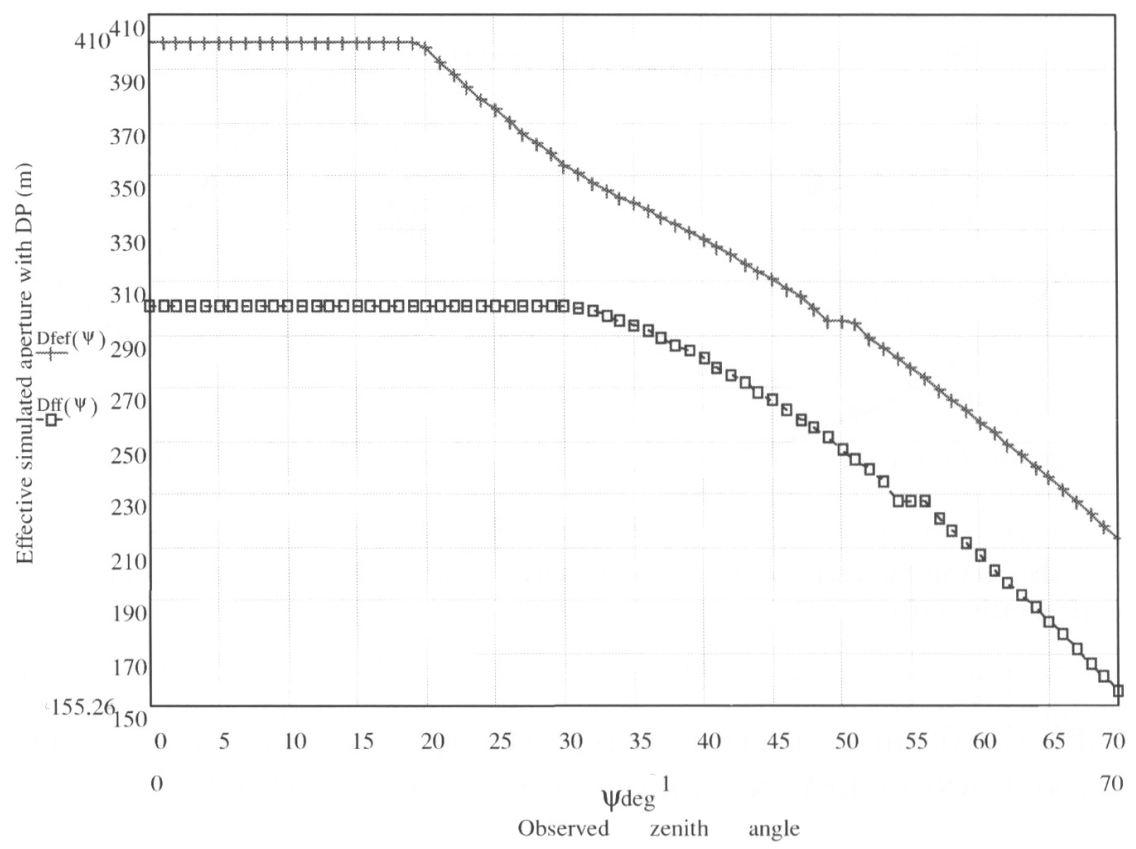

Figure 3. Comparison of available equivalent aperture with only inner $300 \mathrm{~m}$ parabola simulated (lower dashed curve with boxes) and both inner and outer simulated reflector together (upper solid curve with crosses).

Other possible shapes for the outer ring have also been tried successfully, and various, perhaps more important, observational modes are being studied. For instance, we probably could use the different parts (inner and outer) of the aperture, in combination with separate feed systems, to observe different objects, if appropriately scheduled.

\section{Brief Conclusions}

For enlarging the sky coverage of the FAST, a possible choice of the geometric parameters are $\mathrm{R}=300 \mathrm{~m}$, opening angle $=120^{\circ}$ (hence $\mathrm{D}=520 \mathrm{~m}$ ). If the offset illumination and the dual-parabola fitting were finally realized in the project, FAST would be a very powerful radio telescope with maximum observable zenith angle of $70^{\circ}$, minimum equivalent aperture of $200 \mathrm{~m}$, and the potential capability of observing two specified objects at the same time. The beam pattern could also be improved with these measures, with lower sidelobe level and reduced spillover. Consequently, observable declination would range from $-40^{\circ}$ to $+90^{\circ}$, thus including several of the most important molecular-gas-rich galaxies, e.g. NGC253 and M82, and the most important giant molecular clouds and late-type stars in the Galaxy, for fruitful spectral observations. The telescope would also very valuable 
TABLE I

A brief comparison between the FAST and some radio telescopes

\begin{tabular}{lccccccccc}
\hline Telescope & $\theta\left(^{\circ}\right)$ & $\Psi\left(^{\circ}\right)$ & $\mathrm{R}(\mathrm{m})$ & $\mathrm{D}(\mathrm{m})$ & $\mathrm{H}(\mathrm{m})$ & $\mathrm{d}_{\mathrm{e}}(\mathrm{m})$ & $\mathrm{A}_{\mathrm{e}}\left(\mathrm{m}^{2}\right)$ & $\mathrm{S}_{\mathrm{i}}(\%)$ & $\mathrm{S}_{\mathrm{r}}(\%)$ \\
\hline FAST & 120 & 70 & 300 & 519.6 & 150 & 300 & 70686 & 32 & 84 \\
Arecibo & 70 & 20 & 265 & 305 & 48 & 225 & 39635 & 3 & 32.5 \\
GMRT & - & 60 & - & 246 & - & 246 & 47529 & 25 & 82.5 \\
GBT & - & 75 & - & 100 & - & 100 & 7854 & 37.1 & 74 \\
ROT-54 & 180 & 60 & 27 & 54 & 27 & 32 & 804 & 25 & 78.5 \\
\hline
\end{tabular}

for exploration of the SETI 'water hole', and for communicating with space probes. The tracking duration would be not shorter than 8 hours for radio sources with declination of $-15^{\circ}-+85^{\circ}$; this factor is important for VLBI observations and in this respect FAST would be comparable with traditional, fully-steerable parabolic radio telescopes. The spillover and ground-source noise would be further reduced and no screen around the surface would be required, if hills around the depression were 60-70 $\mathrm{m}$ higher than the edge of the main surface.

If the observable zenith angle could be extended to $70^{\circ}$, The FAST would have an 'instantaneous sky coverage' of 32\% of the whole sky, and an 'earth-rotation sky coverage' of $84 \%$ of the whole sky. FAST would also be the most sensitive radio telescope, being 5.4, 2.3 and 1.5 times more sensitive than the VLA, the Arecibo Telescope and GMRT respectively, providing state-of-the-art receivers were utilized.

Finally, a comparison of the main parameters and performances indicators between FAST and some other key radio telescopes is shown in Table I. Here $\theta$ means the opening angle of the spherical telescope, $\Psi$ - the maximum observable zenith angle, $d_{e}$ and $A_{e}$ - the illuminated aperture and area, $S_{i}$ and $S_{r}$ - the instantaneous and the earth-rotation sky coverage, $\mathrm{D}$ - the physical or equivalent aperture of telescopes.

\section{References}

Qiu, Y.H.: 1998, AAS 18, 221.

Wu, S.Y. and Chen, H.S.: 1996, Astrophysics Reports (Publ. B.A.O.) 29, 20.

Wu, S.Y.: 1999, MEMOS of the FAST 1, to be published.

Wu, S.Y.: 1999b, Astrophysics Reports (Publ. B.A.O.) 33, 89.

Wu, S.Y.: 2000, Astrophysics Reports (Publ. B.A.O.) 35, 111. 